\title{
Hand-to-Hand: An Intermanual Illusion of Movement
}

\author{
Dario Pittera \\ Disney Research, University of Sussex \\ Pittsburgh (PA), Brighton (Falmer) \\ USA, UK \\ d.pittera@sussex.ac.uk
}

\author{
Marianna Obrist \\ University of Sussex \\ Brighton, Falmer, UK \\ m.obrist@sussex.ac.uk
}

\author{
Ali Israr \\ Disney Research \\ Pittsburgh, PA, USA \\ israr@disneyresearch.com
}

\begin{abstract}
Apparent tactile motion has been shown to occur across many contiguous part of the body, such as fingers, forearms, and back. A recent study demonstrated the possibility of eliciting the illusion of movement from one hand to the other when interconnected by a tablet. In this paper we explore intermanual apparent tactile motion without any object between them. In a series of psychophysical experiments we determine the control space for generating smooth and consistent motion, using two vibrating handles which we refer to as the Hand-to-Hand vibrotactile device. In a first experiment we investigated the occurrence of the phenomenon (i.e., movement illusion) and the generation of a perceptive model. In a second experiment, based on those results, we investigated the effect of hand postures on the illusion. Finally, in a third experiment we explored two visuo-tactile matching tasks in a multimodal VR setting. Our results can be applied in VR applications with intermanual tactile interactions.
\end{abstract}

\section{CCS CONCEPTS}

- Human-centered computing $\rightarrow$ User studies; Haptic devices;

\section{KEYWORDS}

Tactile Illusions; Intermanual Illusion of Movement; Multisensory Integration; Virtual Reality.

\section{ACM Reference Format:}

Dario Pittera, Marianna Obrist, and Ali Israr. 2017. Hand-to-Hand: An Intermanual Illusion of Movement. In Proceedings of 19th ACM International Conference on Multimodal Interaction (ICMI'17). ACM, New York, NY, USA, 9 pages. https://doi.org/10.1145/3136755.3136777

\section{INTRODUCTION}

Touch constitutes a complex experience $[1,18]$. The everyday action of touching an object is in fact a multifaceted task that consists of an awareness of both the object' substance and structural properties $[24,30,32]$. Due to this complexity, haptic experience designers and developers struggle in fully replicating the tactile sensation needed to achieve realistic and compelling experiences. Hence, tactile rendering in interactive technologies, including virtual and augmented

Permission to make digital or hard copies of all or part of this work for personal or classroom use is granted without fee provided that copies are not made or distributed for profit or commercial advantage and that copies bear this notice and the full citation on the first page. Copyrights for components of this work owned by others than ACM must be honored. Abstracting with credit is permitted. To copy otherwise, or republish, to post on servers or to redistribute to lists, requires prior specific permission and/or a fee. Request permissions from permissions@acm.org.

ICMI'17, November 13-17, 2017, Glasgow, UK

(C) 2017 Association for Computing Machinery.

ACM ISBN 978-1-4503-5543-8/17/11 ..\$15.00

https://doi.org/10.1145/3136755.3136777

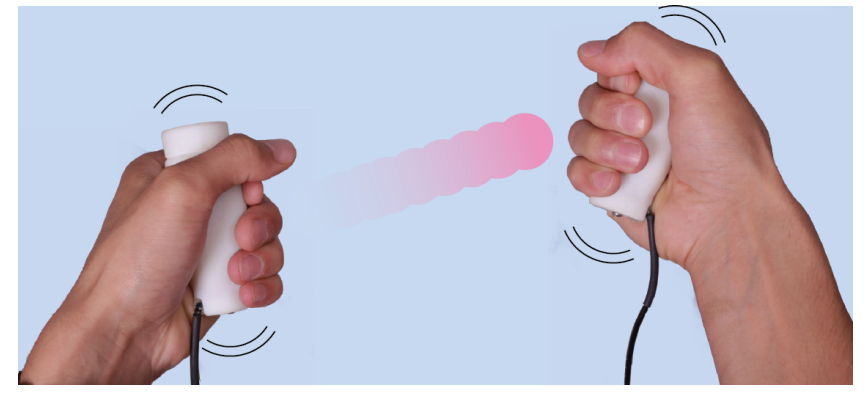

Figure 1: Illustration of the intermanual illusion of movement using the Hand-to-Hand vibrotactile device.

reality, is still limited. Research into tactile illusions provides a relatively simple, technically and computationally economic way of addressing the challenge [9]. The core idea behind tactile illusions is that a tactile sensation can be reproduced convincingly without the need to render every single aspect of the phenomenon [5, 20, 22]. In this paper, we focus particularly on the apparent tactile movement illusion (Figure 2) where two actuators are activated, and the stimulus-onset asynchrony (SOA) is modulated, so that the user will perceive a feeling of movement between two sites of stimulation. Here we present Hand-to-Hand (Figure 1), a vibrotactile illusory movement between two hands. We investigated the possibility of an intermanual illusion of movement without a device connecting the two hands (e.g., holding a tablet), but relying only on a handle held in each hand. In the first experiment, we investigated the feasibility of the illusion and determined the optimal parameters to evoke it. In the second experiment, the effect of postures on the users' perception is examined. Finally, we applied the developed perceptive model to assess users' multimodal integration between touch and vision in a VR setting. The contributions of this paper are threefold: (1) A systematic psychophysical investigation of the occurrence of the apparent tactile movement illusion on non-contiguous and not interconnected parts of the body, which allowed us to determine the parameters for establishing a perceptive model for tactile rendering. (2) Previous studies had shown that the temporal order judgment (TOJ) of a tactile stimuli's onset between two hands may vary according to different postures, in particular, varying the distance between the hands [35]. From the point of view of free-space user interactions, where a user is free to move the limbs in space, the perception of tactile stimuli with varying postures of the arms is important. Therefore, in the second experiment, we investigated different postures of the hands to assess the possible perceptual influence on the model previously established. (3) Lastly, we applied the model in a VR environment to examine the visuo-tactile integration. Overall, our results contribute to a richer understanding of 

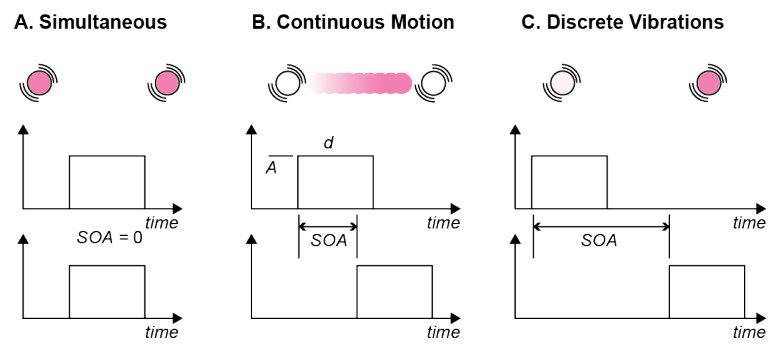

Figure 2: Representation of the tactile illusion of movement, showing the perceptive effect according to different stimulus onset asynchrony (SOA). If the SOA is too long, the perception will result in two discrete vibrations (right). If the SOA is too short, the perception will be merged in a single point (left). With an optimal SOA, a motion will be perceived (middle).

multimodal integration, and will guide designers in their effort to design more immersive, realistic, and even more compelling tactile experiences when interacting with technology.

\section{BACKGROUND}

Tactile feedback was already of interest in late 1957 when a film director, Morton Heilig, conceived one of the first examples of immersive and multisensory technology. His machine, the Sensorama, was able to convey to the spectator tactile and kinesthetic feedback, stereo-sound, 3D vision, and smell [10]. Two years later another film director, William Castle, in his movie "The Tingler", introduced a vibrating chair called Percepto, synchronized with the onscreen projection [12]. Today we have more advanced ways of integrating multiple senses and yet still face various challenges when creating compelling tactile experiences. In this work, we build on prior research on tactile illusions, focusing on creating intermanual tactile sensations.

\subsection{Relevance of Tactile Illusions}

There is a vast interest in the exploration of the sense of touch from a perceptual and technical point of view (for a detailed review on haptic perception, see [24]. Within human-computer interaction, there are numerous examples of tactile devices, each one focused on different aspects of touch, such as: the Maglev levitation haptic interface [2], applying a 6 DOF feedback force; the HapTip [6] and the Skin Stretch Haptic Device [4], using shear force; the Dexmo exoskeleton [7], interacting with the kinesthetic of the hand; and the UltraHaptics mid-air haptic device [3], which employs high frequency mid-air vibrations to create touchless interaction.

With the proliferation of virtual and augmented reality technology, there is also an increased interest in creating more immersive and compelling experiences. Most recent efforts to achieve more realistic experiences are through the integration of tactile stimulation in virtual environments, exemplified by Oculus Touch or the HTC Vive with tactile controllers [8]. Building on those efforts, there is however a lack of understanding of the visuo-tactile interaction and integration that would allow us to design for new interactive experiences. Replicating all tactile sensations would be arduous if not impossible considering the complexity of touch [1,18]. Recent studies illustrated an alternative way to explore touch through exploiting tactile illusions $[9,11,23]$ using a psychophysical approach (i.e., the investigation of the relation between physical stimuli and the perception they create $[13,19]$. These prior works have identified three main types of illusions of movement: 1) the cutaneous rabbit illusion; 2) the haptic funneling; and 3) the apparent tactile movement illusion.

In the first type of tactile illusion, the cutaneous rabbit illusion, two vibrotactile actuators are modulated in a timely fashion to create a third illusory perceptual sensation like a rabbit hopping in between the two real stimulators [5]. In the haptic funneling illusion, two actuators vibrating with different intensity are able to create a third in-between point, whose position will be determined accordingly to the variation of the intensity of the two vibrations [38]. In the apparent tactile movement illusion, two actuators are activated and the stimulus-onset asynchrony (SOA) is modulated so that the user will perceive a feeling of movement between the two sites of stimulation (see Figure 2) [20]. Here, three scenarios are possible: a) if the SOA is too long, the two vibrations will be perceived as discrete and no illusion of movement will occur (Figure 2 , right). b) If the SOA is too short, the two vibrations will be perceptually merged into a single one and no illusion of movement will occur (Figure 2, left). c) Only when the SOA is optimal will the two vibrations appear as movement (Figure 2, middle). For a more extensive overview of tactile illusions see [9, 23].

\subsection{Intermanual Tactile Illusions of Movement}

Prior works mainly focused on delivering the tactile illusion on contiguous parts of the body: on the forearm [22]; on the fingers [28]; and on the back $[13,15]$. A recent study focused on the effect of the illusion of tactile movement between hands while holding a tablet, thereby adding a visual cue on top of the tactile sensation [41]. Moreover, Israr et al. [14] explored the possibility of enhancing gameplay using a glove containing one vibrating actuator to enrich storytelling through tactile feedback [16]. Beyond gaming purposes, tactile feedback had also been considered for simulators [34, 37, 43] learning Reading et al. [31], Santos and De Carvalho [33], and teleoperation systems [17, 26, 27, 29?].

Understanding the visuo-tactile interaction can guide the development of future multimodal applications, in particular VR applications where the integration of tactile sensations can make a real difference with respect to the feeling of presence and the sense of immersion, and it is essential to understand if the illusion can also occur on non-contiguous and not interconnected parts of the body.

\section{THE HAND-TO-HAND DEVICE}

To facilitate the exploration of intermanual tactile illusions of movement for non-contiguous parts of the body (hand to hand) we built a vibrotactile device, we refer to as Hand-to-Hand. The device consists of two 3D printed handles (see Figure 3), each containing a voice coil actuator (www.moticont.com, model GVCM-019-032-02) sandwiched between two springs. The spring stiffness is selected such that the transfer function of the handle is similar to that of the human detection threshold functions [8]. The device is controlled through audio production software (www.puretata.com) and 
interfaced using UDP (User Datagram Protocol). This simple but effective framework allowed us to design a series of experiments where we precisely controlled the delivery of a tactile sensation (i.e., frequency, amplitude, SOA, ramp up/down of the signal) using integrated development environments (IDE) and game engines (Unity) In the third experiment, we attached an additional button to the top of each handle to allow participants to enter their responses.

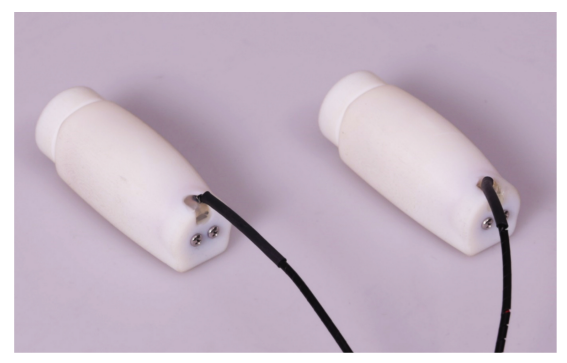

Figure 3: The Hand-to-Hand device consists of two 3D printed handles containing two voice coils sandwiched between springs and controlled by real-time software systems for interactive control.

\section{EXPERIMENT 1: HAND-TO-HAND TACTILE ILLUSION OF MOVEMENT}

The aim of this experiment was firstly to investigate whether the intermanual illusion between the hands occurred when no objects were present between the hands, and secondly, if the illusion occurs, to determine optimal parameters to elicit a smooth illusion of movement. The experiment follows a psychophysical approach that establishes a mathematical model relation between the duration of the stimulation $(\boldsymbol{D})$ and the temporal onsets $(\boldsymbol{S O A})$.

\subsection{Participants}

The study was carried out in a single session with 10 participants ( 6 female, median $=24$ ). They had normal or glasses/lens corrected vision and no history of neurological or psychological disorders. All participants were right-handed. Upon arrival, participants were asked to read the information sheet and sign a consent form, followed by a task explanation. All participants were compensated with US \$10.

\subsection{Experimental Setup}

Participants sat on a chair with arm supports. To control the intermanual distance we created a board to constrain the participants' hand movements. Two areas were marked on the board using foam strips, $2 \mathrm{~cm}$ tall, as boundaries (Figure 4).

\subsection{Methods}

Participants were provided with the Hand-to-Hand device (Figure 3). Before beginning the main experiment, participants had the opportunity to familiarize themselves with the stimuli. In a pilot study with another 10 participants, we determined that the frequency $(70$, 100 or $250 \mathrm{~Hz}$ ) of stimulation did not influence the rating of the smoothness of the illusory motion, however, the duration settings

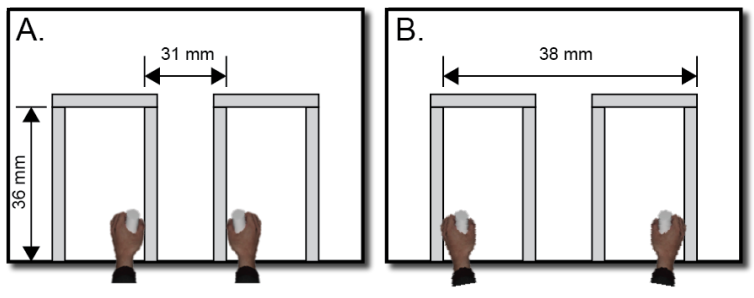

Figure 4: Experiment 1 set up: A) Regular hand posture. B) Wide hand posture.

(100 and $400 \mathrm{~ms}$ ) varied subjective ratings on the smooth motion. Therefore we set the test frequency at $70 \mathrm{~Hz}$. The amplitude was set at $28 \mathrm{~dB}$ SL ( $\mathrm{dB}$ above the detection threshold), to be sure participants could perceive the vibrations distinctly. In addition, we chose two durations (i.e., $\mathrm{D}=100$ and $400 \mathrm{~ms}$ ) based on prior work [42]. For each duration we chose a different set of 7 temporal onset separations, SOA, equally divided as in [42]. For the $100 \mathrm{~ms}$ duration the SOAs ranged from $15 \mathrm{~ms}$ to $160 \mathrm{~ms}$, and for the $400 \mathrm{~ms}$ duration SOAs ranged from $15 \mathrm{~ms}$ to $350 \mathrm{~ms}$. These SOA ranges are required to reach a plausible effect of movement [42]. Every tactile stimulus was set to a linear ramp up and ramp down at a time equal to $20 \%$ of the stimulus duration [42].

Each duration and SOA was tested in two motion directions (left-to-right and right-to-left) and two postures. Participants' arms were comfortably leaning on the chair's arm supports, with the hands resting on the board at $31 \mathrm{~cm}$ distance for the regular posture (Figure 4A), and $38 \mathrm{~cm}$ for the wide posture (Figure 4B). For each duration and posture, participants were also tested in a control condition ( $\mathrm{SOA}=0,12$ times) to account for their random responses. In total, this experiment consisted of 180 trials, three repetitions of 2 duration, 2 direction, 2 posture 7 SOAs +12 control conditions, divided in three blocks of 60 tactile stimuli.

Stimuli were presented in a randomized order one at the time, with at least 5 seconds gap to avoid tactile habituation. After the stimulus was presented, participants were verbally asked if the sensation of movement occurred. In the case of a negative response then the participant's rating was marked ' 0 ' and the next trial was presented. In the case of positive response, the same stimulus was repeated and participants were asked to verbally rate the smoothness of motion on a scale from 1 (discrete) to 7 (continuous). Each block was separated by a 2-minute break. Participants wore headphones to mask environmental and the device sounds. Moreover, a "beep" sound was played through the headphones before the beginning of every trial. Overall, the experiment lasted for 30 minutes.

\subsection{Results}

To ensure that the rating scale was used appropriately, users' ratings ( 0 , no motion, through 7 , continuous motion) were averaged for the two durations across participants. At $\mathrm{SOA}=0$ (catch trials), the overall ratings were 1.68 and 3.2 for $100 \mathrm{~ms}$ and $400 \mathrm{~ms}$, respectively. Figure 5 illustrates the average ratings as a function of SOA for the 2 durations, 2 directions, and 2 postures. The error bars show standard errors of the mean. Each plot was regressed with a best-fit quadratic trend, and the corresponding correlation coefficients are shown 

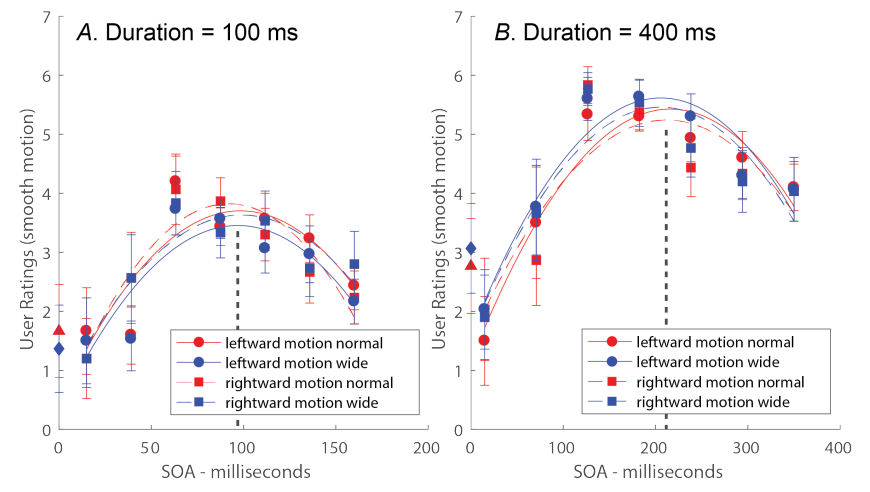

Figure 5: Plots of user ratings of the illusion of movement (y axis) per SOAs (x axis) at A) $100 \mathrm{~ms}$ and B) $400 \mathrm{~ms}$.

in Table 1. The two lowest parts of the curves correspond to low SOAs (merged tactile perception) and to high SOAs (discrete tactile perception). The peaks of the curves correspond to the optimal values of SOAs and are reported in Table 1.

We checked the rating scores divided per stimulus' duration, and found that the data significantly deviates from a normal distribution. Therefore, we proceeded using a Friedman test. Results show a significant difference between the two durations, $\chi^{2}(1)=183.95$, $\mathrm{p}<0.001$. Looking at Figure 5, it is clear that the $400 \mathrm{~ms}$ duration (right) has a more powerful effect. That is, the illusion of movement is perceived strongly. The different SOA resulted significantly different as well, $\chi^{2}(6)=143.59, \mathrm{p}<0.001$ for $100 \mathrm{~ms}$, and $\chi^{2}(6)=99.56$, $\mathrm{p}<0.001$ for $400 \mathrm{~ms}$.

When analysing the direction of vibrations, we did not find an effect, $\chi^{2}(1)=0.214, \mathrm{p}=0.64$ for $100 \mathrm{~ms}$ and $\chi^{2}(1)=1.667, \mathrm{p}=0.2$ for $400 \mathrm{~ms}$. The posture of the hands did not result in a significant difference, $\chi^{2}(1)=0.44, \mathrm{p}=0.51$ for $100 \mathrm{~ms}$ and $\chi^{2}(1)=0.68, \mathrm{p}=0.41$ for $400 \mathrm{~ms}$. In conclusion, our results show that the duration and SOA are the only significant parameters with an effect on the illusion of movement, confirming previous works [42]. Fitting the peaks data in Table 1 into a regression model, resulted in the following model:

$$
y=0.38 x+58.8, R^{2}=.99
$$

where, $\mathrm{x}$ is representing the duration of the stimulus in milliseconds and $y$ is the optimal SOA in milliseconds related to that specific duration.

Table 1: The optimal values of SOA for the eight curves, along with the quadratic fit $\left(\mathbf{R}^{2}\right)$.

\begin{tabular}{lcl}
\hline Stimulus & Peak & R2 \\
\hline $100 \mathrm{~ms}$ - regular posture, rightward motion & 98.31 & 0.69 \\
$100 \mathrm{~ms}$ - regular posture, leftward motion & 92.00 & 0.87 \\
$100 \mathrm{~ms}$ - wide posture, rightward motion & 96.82 & 0.75 \\
$100 \mathrm{~ms}$ - wide posture, leftward motion & 98.85 & 0.82 \\
$400 \mathrm{~ms}$ - regular posture, rightward motion & 215.99 & 0.92 \\
$400 \mathrm{~ms}$ - regular posture, leftward motion & 211.87 & 0.73 \\
$400 \mathrm{~ms}$ - wide posture, rightward motion & 205.75 & 0.91 \\
$400 \mathrm{~ms}$ - wide posture, leftward motion & 205.00 & 0.83 \\
\hline
\end{tabular}

\section{EXPERIMENT 2: TEMPORAL ORDER JUDGMENT TASK (TOJ)}

The illusion of movement is the result of an implicit temporal order judgment (TOJ) between the two stimuli perceived on the hands. The aim of this second experiment was to explore whether changing the posture of the arms (see Figure 6) influences the users perception and temporal judgment. In fact, prior works suggest that changing the posture of the arms could affect the temporal judgment of the two stimuli [35], and consequently could also affect the perception of the illusion of intermanual movement. If an effect was observed, it would have been used to redefine the model established in experiment 1 .

\subsection{Pilot Study and Participants}

To explore and choose the device's settings we conducted a pilot study with 12 participants ( 4 female, median $=24.5$ ). They had normal or glasses/lens corrected vision and no history of neurological or psychological disorders. All participants were right-handed. Upon arrival, participants were asked to read the information sheet and sign a consent form, followed by a task explanation. All participants were compensated with US $\$ 10$.

\subsection{Experimental Setup}

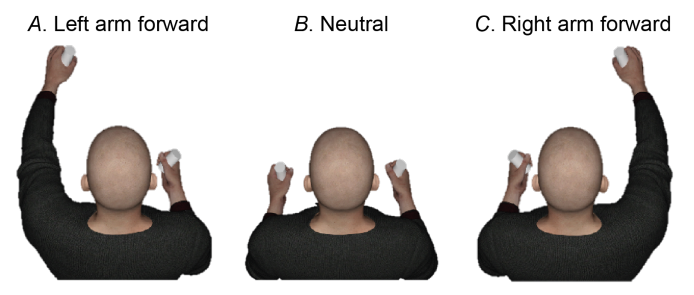

Figure 6: Experiment 2 set up including three different postures: left arm forward, neutral, right arm forward.

The experimental setting used for this pilot study was the same as in Experiment 1, with the difference being that participants were tested on a temporal order judgment (TOJ) task. In other words, participants had to answer which of the two handles vibrated first. Since participants had to hold the two handles, we provided them with a 4 buttons foot pedal (Olympus America Inc., model RS31H) to enter their response.

\subsection{Methods}

For the tactile stimuli we used one frequency $(70 \mathrm{~Hz})$, two durations (100 and $400 \mathrm{~ms}$ ) and a set of 11 SOAs (from $-100 \mathrm{~ms}$ to a $100 \mathrm{~ms}$ in $20 \mathrm{~ms}$ increments). Positive SOA corresponded to the left handle vibrated first, and the negative SOA corresponded to the right handle vibrated first. The stimuli's ramp up and down time was kept at $20 \%$ of the stimulus duration. The amplitude of the signal frequency was $18 \mathrm{~dB}$ SL. Participants were required to switch posture of the arms in three possible ways (Figure 6): regular posture (as in Experiment 1), left arm completely extended in front of the shoulders and the right arm in neutral posture (condition left forward), or vice-versa (right forward). 
We controlled the distance between participants' hands again using the board in Figure 4. A picture showing the testing posture appeared on the interface before the trial started. Participants had to press the central button of the pedal to play the stimulus. Stimuli could start from the left or from the right hand. After the stimulation, the participant had to indicate which hand they felt was stimulated first; pressing the left button on the pedal if the left handle vibrated first and vice-versa for the right side. After they answered, another posture followed and the entire procedure was repeated until the end of the block of 33 stimuli. Participants wore headphones to cover the environmental and the device noises. Each stimulus was repeated three times for a total of 198 trials $(3 \times 2$ duration $\times 3$ postures $\mathrm{x} 11 \mathrm{SOA}$ ). Overall, the experiment lasted for one hour.

\subsection{Results}
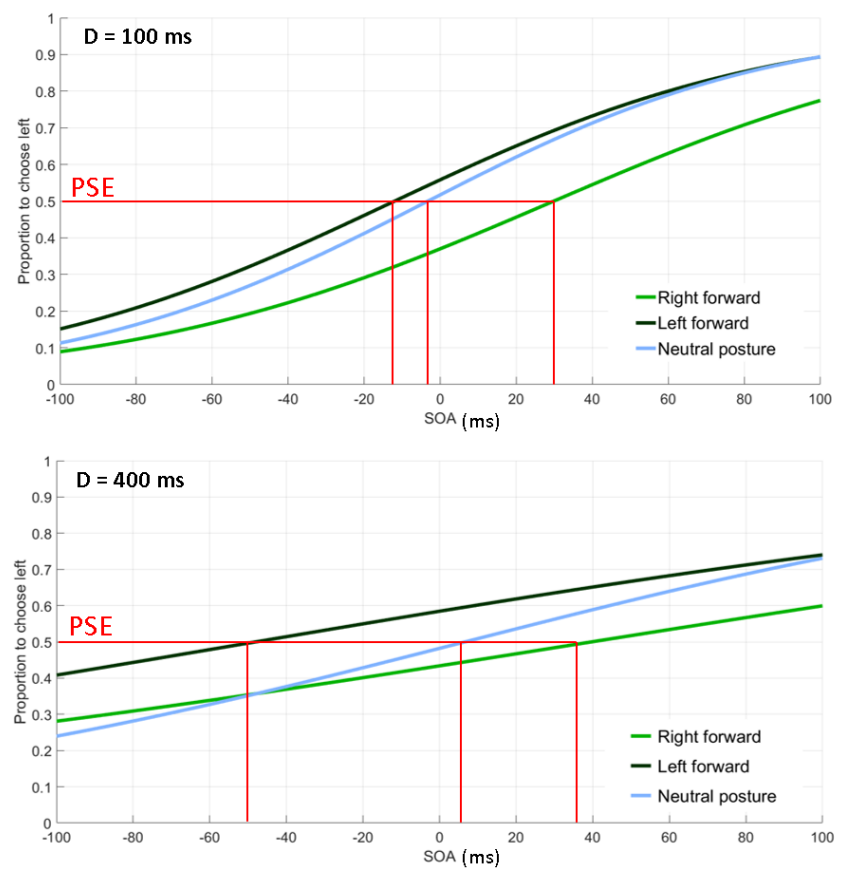

Figure 7: The three cumulative Gaussian functions for duration equal to $100 \mathrm{~ms}$ (top), and $400 \mathrm{~ms}$ (bottom). On the $y$ axis, the probability of choosing left as the first vibrating. On the $x$ axis, the SOAs: negative values correspond to the vibrations starting from right.

Figure 7 shows the three psychophysics curves resulting from the data collection, for $d=100 \mathrm{~ms}$ and $\mathrm{d}=400 \mathrm{~ms}$. Each curve corresponds to a posture. The SOAs values appear on the $\mathrm{x}$ axis: negative if the direction was going from right to left, positive when the vibrations were starting from the left handle. The probability of selecting the left handle as the first vibration is plotted on the y axis. In a psychophysics curve, the point of subjective equality (PSE) indicates the point at which one can no longer perceive two stimuli as distinct. In our case, it is the point where participants could not feel which vibration was coming first, either the right or the left one. For $\mathrm{d}=100 \mathrm{~ms}$, the green curve appears shifted to the right.
An ANOVA repeated measures showed a non-significant difference between the three postures $(\mathrm{F}(2,20)=3.11, \mathrm{p}=0.07)$, although with a low p-value. For $\mathrm{d}=400 \mathrm{~ms}$, data were not following a normal distribution. We used a Friedman test to test differences between the three curves. The test did not show any significant difference between the three postures, $\left(\chi^{2}(2)=5.09, p=0.08\right)$, and also in this case the p-value appeared low.

These results suggest that the posture did not clearly influence participant's performance in the TOJ task, but there might be a tendency in doing that. With the amplitude chosen participants reported uncertainty regarding the occurrence of both the vibrations, and this could explain the noisy distributions of data in Figure 7. Hence, we conducted another study with a higher amplitude equal to $28 \mathrm{~dB}$ SL to ensure a clear vibrotactile perception. We chose only one duration $(100 \mathrm{~ms})$ and repeated each stimulus 7 times instead of three to get more robust data.

\subsection{Main Study and Participants}

The study was carried out in a single session by 10 participants ( 8 female, median age $=22.5$ years old). They had normal or glasses $/$ lens corrected vision and no history of neurological or psychological disorders. All participants were right-handed. Upon arrival, participants were asked to read the information sheet and sign a consent form, followed by a task explanation. All participants were compensated with US $\$ 5$.

\subsection{Experimental Setup}

The experimental setting was same as in the pilot study (see Section 5.1.1).

\subsection{Methods}

For this experiment we followed the same procedure used in the pilot study (see Section 5.1.2) with the only difference being we had one vibration's duration (100 ms) at an amplitude of $28 \mathrm{~dB}$ SL. This experiment consisted of 33 stimuli repeated three times in three postures (99 trials). Participants wore headphones to cover the environmental and the device noises. Overall, the experiment lasted for 30 minutes.

\subsection{Results}

Figure 8 shows three psychophysics curves resulting from the data collection. Each curve corresponds to a posture. The SOAs values appear on the $\mathrm{x}$ axis: negative values refer to the direction going from right to left, positive when the vibrations were starting from the left handle. The probability of selecting the left handle as the first vibration is plotted on the y axis. In a psychophysics curve, the point of subjective equality (PSE) indicates the point at which one can no longer perceive two stimuli as distinct. In our case, it is the point where participants could not feel which vibration was coming first, either the right or the left one.

The three curves in Figure 8 appear to be very similar. Our data were following a normal distribution and no outliers were found. An ANOVA repeated measures performed on the 10 participants PSE data confirmed a non-significant difference between the three curves, $\mathrm{F}(2,18)=2.595, \mathrm{p}=0.102$. Therefore, our results suggest that the posture does not influence participant's performance in the TOJ 


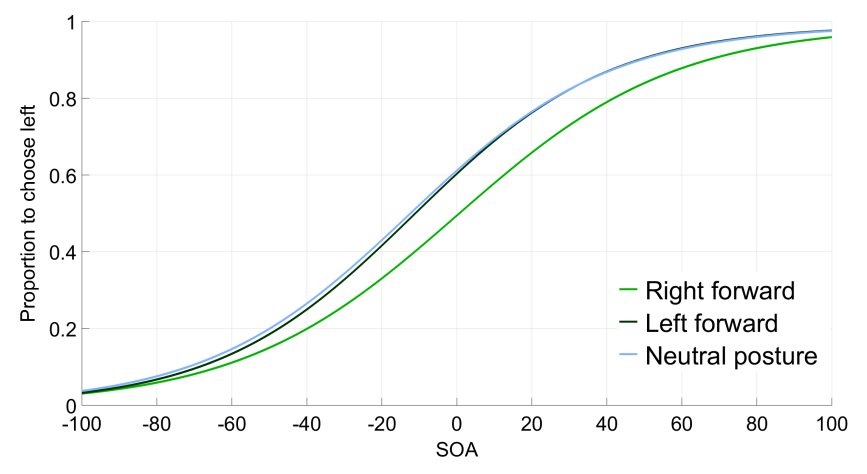

Figure 8: The three cumulative Gaussian functions. On the $y$ axis, the probability of choosing left as the first vibrating. On the $x$ axis, the SOAs: negative values correspond to the vibrations starting from right.

task and thus has no crucial effect on the perception of the illusion of movement.

Looking at Figure 8 it is possible to infer that when the SOA was equal to 0 , participants' answers were random, as expected. For very high SOAs instead, the probability of a correct answer (i.e., 'the left hand vibrated first when the left was vibrating first') was almost $100 \%$, as indicated by the saturation of the curve on the value equal to 1 . The threshold was set at $75 \%$ and computed pairing the three curves, and corresponded to a SOA equal to 66.4 ms.

\section{EXPERIMENT 3: VISUAL-TACTILE INTEGRATION IN VIRTUAL REALITY}

With the two previous experiments we investigated the optimal parameters for the illusion of movement and defined a perceptive model that is not affected by the posture. In a final third experiment, we programmed an application that exploits the illusion of intermanual movement in VR, using our established model. Moreover, we are interested in investigating the perceptual integration of visuo-tactile stimuli.

\subsection{Participants}

The experiment was carried out in a single session by a new pool of 10 participants $(6$ female, median $=21)$. They had normal or glasses/lens corrected vision and no history of neurological or psychological disorders. All participants were right-handed. Upon arrival, participants were asked to read the information sheet and sign a consent form, followed by a task explanation. All participants were compensated with $\$ 10$. Six participants could not complete the whole set of trials within the given time of 60 minutes. In total, we collected 692 trials out of 800 .

\subsection{Experimental Setup}

We created a virtual environment (VE) using Unity 3D to investigate the synchronization between tactile and visual stimuli. Participants wore an Oculus DK2 VR headset (960 x 1080 per eye, ca. $75 \mathrm{~Hz}$, $100^{\circ} \mathrm{FoW}$ ), and they could see in the VE two hands attached to a body, while sitting on a chair in front of a desk (see Figure 9). To
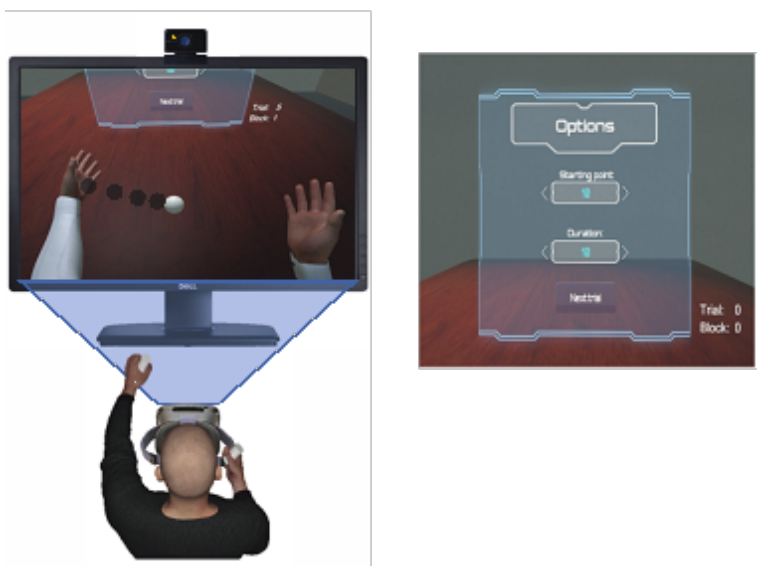

Figure 9: Experiment 3 setup. Participants wore an HMD for VR. In the VE they could see two hands, changing in posture according to the different parameters of the experiment, a body attached, and a white ball moving from one hand to the other at different speeds. (Right) The interface used in VR to complete the matching tasks.

navigate the VR interface, participants were provided with a pedal. The Hand-to-Hand device with the two buttons on the top allowed them to select the different settings of the interface and to skip to the next trial as follows.

\subsection{Method}

During the experiment, the posture of the two hands was visually adjusted in the VE to create the four conditions: regular, wide, left forward and right forward posture (see Figure 9). In practice, at the beginning of the trial, participants could see the VE and they had to adjust their arms' posture to match the ones in VR. The posture also appeared written on the screen in the VE to avoid misunderstanding. The visual stimulus was represented by a white ball moving from one hand to the other at five different speeds (2, $4.5,7,9.5$ and $12 \mathrm{~m} / \mathrm{s}$ for a total of $90,120,170,250,570 \mathrm{~ms}$ for the regular posture, and 110,140, 200, 300 and $680 \mathrm{~ms}$ for the rest of the postures).

We controlled and counter-balanced the direction of the ball (left to right and right to left). At the beginning of every trial, an arrow was indicating the initial hand from which the ball would move from. The tactile stimulus was rendered through the two 3D printed vibrotactile handles used in the previous experiments. This time, we added two buttons on the top of the handles, to allow participants to interact with the VR interface fixed in front of the participants' point of view (see Figure 9).

To navigate, participants were provided with the same pedal as in experiment 2; pressing the central button to select the different options shown in Figure 9. They had the possibility of changing two settings: they could increase or reduce the delay of the onset time of the vibration on the first hand (starting point in Figure 10) and they could extend or shorten the total duration of the tactile event (duration in Figure 10), intended as the SOA plus the duration 

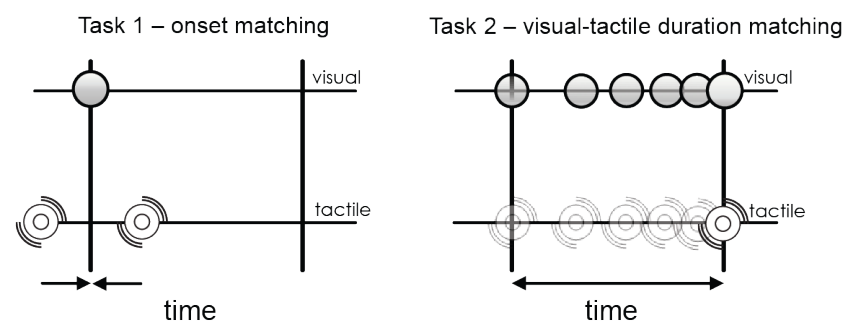

Figure 10: The two matching tasks used in Experiment 3. Task 1) Participants had to match the onset of the visual and tactile stimuli. Task 2) Participants had to match the duration of the visual and tactile stimuli.

of the two vibrations (including the time for the signal to rump up and down). Participants had to complete two tasks:

Task 1: the aim of the first task was to match the visual and the tactile cue onset (Figure 10 left). The visual cue (the white ball) was always visible for a fixed amount of time depending on the speed. Instead, the first vibration on the hand was randomly selected Âs $50 \mathrm{~ms}$ respect to the visual cue. When the tactile stimulus appeared before the visual one, participants had to press the right button on the handle, increasing the stimulus delay by $5 \mathrm{~ms}$. Conversely, if the first vibration was starting after the visual cue, participants had to press the left button on the handle, reducing the delay of the tactile cue by $5 \mathrm{~ms}$. Every time that participants pressed one of the two buttons, the trial was restarted with the new values.

Task 2: The second task consisted of matching the total duration of the two events, the tactile and the visual one. Once participants completed task 1, they had to match the ending point of the two stimuli (Figure 10, right). When the ball was disappearing (once it reached the second hand), also the second vibration (on the second hand) had to disappear. If the tactile stimulus' duration (intended as the duration of the first vibration, plus the SOA, plus the second vibration) was shorter than the visual one, participants had to press the button on the right handle to extend the total tactile duration by $30 \mathrm{~ms}$.

Vice-versa, if the total tactile duration was too long, participants had to press the button on the left handle, shortening the total duration by $30 \mathrm{~ms}$. Also in this case, pressing a button meant restarting the stimulus from the first hand with the new values. The duration of the tactile stimulus was randomly selected between $100 \mathrm{~ms}$ and $400 \mathrm{~ms}$, with the SOA changing in consequence of the duration's value, according to the model obtain from experiment $1(\mathrm{y}=0.38 \mathrm{x}$ +58.8 ).

This experiment consisted of two blocks of 60 randomized stimuli. For the tactile stimuli we used one frequency $(70 \mathrm{~Hz})$ and the duration and SOA were varying according to the model of experiment 1 . The stimuli's ramp up and down time, was kept at $20 \%$ of the stimulus duration. The amplitude of the signal frequency was 28 dBSL.

We used the same board as from experiment 1 and 2 to control participants hands' distance (Figure 4). In total, participants had to complete two blocks of 40 trials in one hour.

\subsection{Results}

The data for Task 1 was negatively skewed, hence we normalized the data using the formula: $\lg 10$ (max value - value). We then analyzed the data using a two-way ANOVA repeated measures test, with speed and posture as factors. The results shows a non-significant effect neither of postures $(\mathrm{F}(3,72)=1.131, \mathrm{p}=.342)$ nor speed $(\mathrm{F}(4,96)$ $=.774, \mathrm{p}=.545)$. Their interaction was also not significant $(\mathrm{F}(12,288)$ $=.440, \mathrm{p}=.946)$. The data for Task 2 was also negatively skewed, hence, we normalized the data before proceeding with the analysis. We again performed a two-way ANOVA repeated measures test with speed and posture as factors.

The results indicate a significant main effect of speed, $F(4,96)=$ $8.585, \mathrm{p}<.001$, and posture, $\mathrm{F}(3,72)=7.173, \mathrm{p}<.001$. The pairwise comparisons between the visual minus tactile duration for specific speeds indicate that the visuo-tactile deltas' scores for the speed of 2 $\mathrm{m} / \mathrm{s}$ and $4.5 \mathrm{~m} / \mathrm{s}$ were different from all of the other speeds $(\mathrm{p}<.001)$, meanwhile the other three speeds were not significantly different from each other $(p>0.05)$. The right forward posture was the only one to differ from the others $(\mathrm{p}<.05)$. The interaction between posture and speed was significant as well, $\mathrm{F}(12,288)=11.053, \mathrm{p}<$ .001 . To further investigate this interaction, we analyzed the simple effects. In particular, it appears that for speed equal to $4.5 \mathrm{~m} / \mathrm{s}$, in the right forward posture, the tactile cue need a shorter duration to be perceived as matching the visual cue $(p=0.048)$. The same is valid for speed equal to $7 \mathrm{~m} / \mathrm{s}(\mathrm{p}=0.019)$.

Finally, for speed equal to $12 \mathrm{~m} / \mathrm{s}$, the posture right forward seems to significantly differ for all the other postures $(p<0.001)$. Figure 11 illustrates the linear fit of our data (the red lines) compared with the 1:1 uncompressed visuo-tactile relation (the black lines). For the regular posture $(31 \mathrm{~cm}$ distance between the two hands) it appears that the tactile duration is compressed with respect to the visual one by a factor of approximately $1 / 4$, meaning that even if the visual duration is increasing, the tactual duration is not increasing accordingly. This is especially true for the wide and right forward posture, where the red line appears to be almost flat. The left forward posture instead, indicates an inverse tendency: the slower the visual stimulus is (long visual duration), the shorter the tactile stimulus is perceived.

\section{DISCUSSION}

Here, we investigated the occurrence of the tactile illusion of movement and its particularities as well as the effects of the postures of the hand, and when integrated in a virtual environment. With the first experiment we established that it is possible to elicit an illusion of movement using tactile stimuli delivered on two hands that are not interconnected by any means, such as a tablet used in prior research [42]. Based on that initial step, we then determined and described the optimal parameters to achieve a smooth tactile illusion of movement using a psychophysical approach.

We generated a perceptual model that expresses the relation between duration and SOA of the tactile stimuli: $y=0.38 \mathrm{x}+58.8$. This model, specifies the optimal parameters to use for achieving a smooth illusion of motion between the hands. In short, the most relevant variables impacting users' perception are duration and the stimulus-onset asynchrony (SOA) of the tactile stimuli, confirming previous results [42]. To understand whether the position of the 


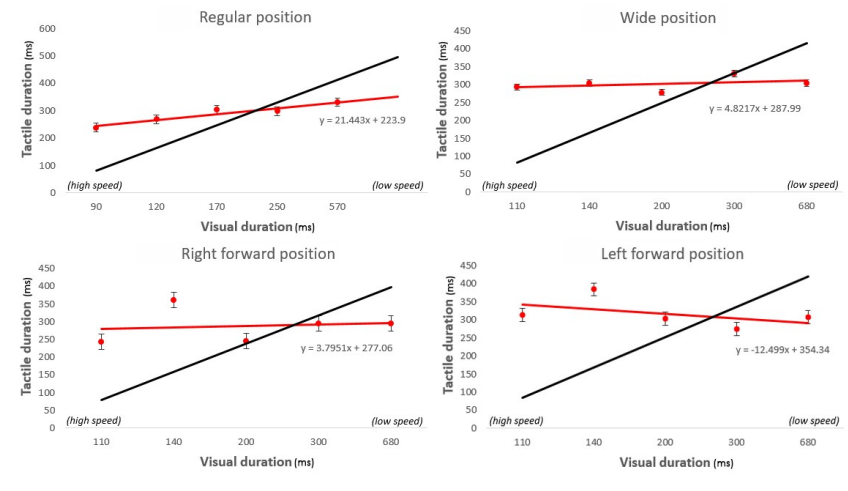

Figure 11: Linear fits of visual and tactile duration, divided per postures (regular, wide, right and left forward). The black line represents the 1:1 uncompressed visuo-tactile relation. The red line represents the linear fit of our data.

users' arms (i.e., posture) influences the temporal perception of the two tactile stimuli, we used a temporal order judgment task (TOJ). This is important because judging the movement means ultimately recognizing which stimulus occur first and hence can guide design decisions in an interactive system based on touch.

Our results showed that the posture does not have any effect on the perception of movement, which is in contrast to prior findings presented by Shore [35]. The difference could be explained based on the use of different SOAs, which are also different to the work presented by Siyan [42]. In particular, the SOAs used in Shore, were $10 \mathrm{~ms}, 30 \mathrm{~ms}, 55 \mathrm{~ms}, 90 \mathrm{~ms}$ and $200 \mathrm{~ms}$ and no indication about the amplitude is provided, which might have a key role in the different results obtained. In fact, in our pilot study, where we used a lower amplitude of about $18 \mathrm{dBSL}$, the results had a different trend, near the significance level. Another difference was the modality of delivering the tactile stimuli, on the finger vs. through a vibrotactile handle. We initially thought that the manual laterality of the participants could have had an effect on the outcome. Our participants were all right-handed, hence, it is hard to answer this question. It is known that crossing the hands has an impact on the TOJ of tactile stimuli $[36,40]$. On the contrary, we found only one study investigating the effect of the hands' distance (not crossed) on a TOJ task [21], other than [35]. In Kuroki's study [21], authors demonstrated how the spatiotopic distance does not influence participants' performance in a simultaneity judgment task, similarly to our results.

In a final step, we programmed a virtual environment, where participants were able to perceive tactile stimuli integrated with visual stimuli in order to assess our perceptive model. In addition we also investigate the visuo-tactile integration in a specific application context, relevant for exploiting tactile illusions and multimodal interaction. The results as summarized in the previous section do not allow clear conclusions. However, one could speculate that for slower velocities, the tactile cue is perceived before the visual one. What we know for certain is that there are no negative visuo-tactile duration deltas (visual delay minus tactile delay). In other words, the tactile stimulus is never happening after the visual cue, as the tactile perception is faster than the visual one. One possible explanation could be that the task used during the exposure phase resulted in an attentional bias towards the tactile modality. According to the 'law of prior entry', attending to one sensory modality speeds up the processing of stimuli in that modality [39], resulting in a change in the PSS (point of subjective simultaneity).

\section{LIMITATIONS}

This work is a first step into the analysis of the tactile illusion of movement without any object in between the two hands. Although our results are promising, we also need to acknowledge some limitations.

One limitation is that participants' hands were static in our experiments, which does not reflect users' behavior in a virtual environment where they move around a space and interact with objects. In order to establish our perceptual model it was necessary to control the movement. However, future work can take users movement into account by creating a more interactive scenario, and consequently also extend our perceptive model. Moreover, future investigations of kinesthetic cues only (with no visual feedback) can now be explored.

Another limitation are the sample sizes in our studies. With a larger number of participants some of the effects (e.g., posture in experiment 2) could become significant, although we consider that unlikely based on our repeated tests in preparation for our studies. Yet, it will be important that future work verifies our findings to strengthen our model and in particular considers any potential bias in the temporal order judgment task towards the right hand.

Finally, we are using a specific actuation technology and focusing on one specific type of illusion. Future work should consider other sensory illusions such as the phantom tactile sensation and sensory saltation, as well as explore the different types of illusions of movements (i.e., cutaneous rabbit illusion, the haptic funneling, and the apparent tactile movement illusion) with other tactile devices and technologies entering the realm of virtual reality (e.g., mid-air touch [25]).

\section{CONCLUSION}

The findings from our three experiments demonstrate that eliciting a sense of illusory movement between two hands is not limited to situations when holding an object with both hands, or across two contiguous parts of the body. This work sets the stage for future investigations of tactile experiences exploiting tactile illusions. Findings with respect to the visuo-tactile integration require additional validation, however the findings are promising with respect to the temporal perception and consequently the design of applications in virtual environments and beyond.

\section{ACKNOWLEDGMENTS}

This project has been funded by Disney Research Pittsburgh, Pittsburgh, US, and partly by the European Research Council (ERC) under the European Union's Horizon 2020 research and innovation program under grant agreement No 638605. We would like to thank Dr. Elia Gatti for his advice and support, and Anthony Trory and Robert Cobden for their valuable comments on the manuscript. 


\section{REFERENCES}

[1] Wouter M Bergmann Tiest and Astrid M L Kappers. [n. d.]. Analysis of haptic perception of materials by multidimensional scaling and physical measurements of roughness and compressibility. ([n. d.]). http://citeseerx.ist.psu.edu/viewdoc/ download?doi=10.1.1.464.4561\&rep=rep1\&type $=$ pdf

[2] P. J. Berkelman and R. L. Hollis. 2000. Lorentz Magnetic Levitation for Haptic Interaction: Device Design, Performance, and Integration with Physical Simulations. The International fournal of Robotics Research 19, 7 (2000), 644-667. https://doi.org/10.1177/027836490001900703

[3] Tom Carter, Sue Ann Seah, Benjamin Long, Bruce Drinkwater, and Sriram Subramanian. 2013. UltraHaptics : Multi-Point Mid-Air Haptic Feedback for Touch Surfaces. Proc. UIST 2013 (2013), 505-514. https://doi.org/10.1145/2501988.2502018

[4] Francesco Chinello, Claudio Pacchierotti, Nikos G Tsagarakis, and Domenico Prattichizzo. [n. d.]. Design of a Wearable Skin Stretch Cutaneous Device for the Upper Limb. ([n. d.]). http://sirslab.dii.unisi.it/papers/2016/ChPaTsPr-HAPTICS16.pdf

[5] Frank A. Geldard and Carl E. Sherrick. 1972. The cutaneous "rabbit": a perceptual illusion. Science (New York, N.Y.) 178, 57 (1972), 178-179. https://doi.org/10.1126/ science.178.4057.178

[6] Adrien Girard, Maud Marchal, Florian Gosselin, Anthony Chabrier, FranÃ ğois Louveau, and Anatole Lécuyer. 2016. HapTip: Displaying Haptic Shear Forces at the Fingertips for Multi-Finger Interaction in Virtual Environments. Frontiers in ICT 3 (4 2016), 6. https://doi.org/10.3389/fict.2016.00006

[7] Xiaochi Gu, Yifei Zhang, Weize Sun, Yuanzhe Bian, Dao Zhou, and Per Ola Kristensson. 2016. Dexmo: An Inexpensive and Lightweight Mechanical Exoskeleton for Motion Capture and Force Feedback in VR. In Proceedings of the $2016 \mathrm{CHI}$ Conference on Human Factors in Computing Systems - CHI '16. ACM Press, New York, New York, USA, 1991-1995. https://doi.org/10.1145/2858036.2858487

[8] Nobuhisa Hanamitsu and Ali Israr. 2011. Haplug : A Haptic Plug for Dynamic VR Interactions Haptic Plug. 432, Lvcm (2011), 479-483. https://doi.org/10.1007/ 978-981-10-4157-0\{_\}80

[9] Vincent Hayward. 2008. A brief taxonomy of tactile illusions and demonstrations that can be done in a hardware store. Brain Research Bulletin 75, 6 (2008), 742-752. https://doi.org/10.1016/j.brainresbull.2008.01.008

[10] Morton L Heilig. 1962. Sensorama simulator. (1962), 50 pages. http://patimg2.uspto.gov/.piw?docid=03050870\&PageNum=9\&IDKey= 3551C1D945D5\&HomeUrl=http://patft.uspto.gov/netacgi/nph-Parser?Sect1= PTO2\%252526Sect2=HITOFF\%252526p=1\%252526u=\%25

[11] N. Hogan, B.A. Kay, E.D. Fasse, and F.A. Mussa-Ivaldi. 1990. Haptic Illusions Experiments on Human Manipulation and Perception of \&quot;Virtual Objects\&quot;. Cold Spring Harbor Symposia on Quantitative Biology 55, 0 (1 1990), 925-931. https://doi.org/10.1101/SQB.1990.055.01.086

[12] Wijnand Ijsselsteijn. 2003. Presence in the Past: what can we learn from Media History? Being There: Concepts, effects and measurement of user presence in synthetic environments (2003), 24.

[13] Ali Israr and Ivan Poupyrev. 2011. Tactile Brush : Drawing on Skin with a Tactile Grid Display. Proceedings of CHI'11 (2011), 2019-2028. https://doi.org/10.1145/ 1978942.1979235

[14] Ali Israr, Siyan Zhao, Kyna McIntosh, Jaekyun Kang, Zachary Schwemler, Eric Brockmeyer, Mark Baskinger, and Moshe Mahler. 2015. Po2 : Augmented Haptics for Interactive Gameplay. ACM SIGGRAPH 2015 Emerging Technologies (2015), 2028. https://doi.org/10.1145/2782782.2792489

[15] Ali Israr, Siyan Zhao, Kyna Mcintosh, Zachary Schwemler, Adam Fritz, John Mars, Job Bedford, Christian Frisson, Ivan Huerta, Maggie Kosek, Babis Koniaris, and Kenny Mitchell. 2016. Stereohaptics : A Haptic Interaction Toolkit for Tangible Virtual Experiences. (2016), 2-3.

[16] Ali Israr, Siyan Zhao, Kaitlyn Schwalje, Roberta L. Klatzky, and Jill Lehman. 2014 Feel Effects: Enriching Storytelling with Haptic Feedback. ACM Transactions on Applied Perception 11, 3 (2014), 1-17. https://doi.org/10.1145/0000000.0000000

[17] Seiichiro Katsura, Wataru Iida, and Kouhei Ohnishi. 2005. Medical mechatronics An application to haptic forceps. Annual Reviews in Control 29, 2 (2005), 237-245. https://doi.org/10.1016/j.arcontrol.2005.05.003

[18] David Katz and Lester E. Krueger. [n. d.]. World of touch. 260 pages.

[19] Frederick Kingdom and Nicolaas Prins. 2013. Psychophysics: A Practical Introduction. Vol. 53. 1689-1699 pages. https://doi.org/10.1017/CBO9781107415324.004

[20] Jacob H Kirman. 1974. Tactile apparent movement: The effects of interstimulus onset interval and stimulus duration. Perception \& Psychophysics 15, 1 (1974) 1-6.

[21] Shinobu Kuroki, Junji Watanabe, Naoki Kawakami, Susumu Tachi, and Shin'Ya Nishida. 2010. Somatotopic dominance in tactile temporal processing. Experimental Brain Research 203, 1 (2010), 51-62. https://doi.org/10.1007/s00221-010-2212-8
[22] Eugene C. Lechelt and Richard Borchert. 1977. The interdependence of time and space in somesthesis: The Tau effect reexamined. Bulletin of the Psychonomic Society 10, 3 (9 1977), 191-193. https://doi.org/10.3758/BF03329320

[23] Susan J. Lederman and Lynette a. Jones. 2011. Tactile and Haptic Illusions. IEEE Transactions on Haptics 4, 4 (2011), 273-294. https://doi.org/10.1109/TOH.2011.2

[24] S. J. Lederman and R. L. Klatzky. 2009. Haptic perception: A tutorial. Attention, perception \& psychophysics 71, 3 (2009), 481-489. https://doi.org/10.3758/APP

[25] Benjamin Long, Sue Ann Seah, Tom Carter, and Sriram Subramanian. 2014. Rendering volumetric haptic shapes in mid-air using ultrasound. ACM Transactions on Graphics 33, 6 (2014), 1-10. https://doi.org/10.1145/2661229.2661257

[26] Steven Martin and Nick Hillier. 2009. Characterisation of the Novint Falcon Haptic Device for Application as a Robot Manipulator. Australasian Conference on Robotics and Automation (ACRA) (2009), 1-9.

[27] Hermann Mayer, Istvan Nagy, Alois Knoll, Eva U Braun, Robert Bauernschmitt, and RÃijdiger Lange. 2007. Haptic Feedback in a Telepresence System for Endoscopic Heart Surgery. Presence: Teleoperators and Virtual Environments 16, 5 (10 2007), 459-470. https://doi.org/10.1162/pres.16.5.459

[28] Makoto Miyazaki, Masaya Hirashima, and Daichi Nozaki. 2010. The "Cutaneous Rabbit" Hopping out of the Body. Fournal of Neuroscience 30, 5 (2010), 1856-1860. https://doi.org/10.1523/JNEUROSCI.3887-09.2010

[29] Jaeheung Park. 2006. A Haptic Teleoperation Approach Based on Contact Force Control. The International fournal of Robotics Research 25, 5-6 (5 2006), 575-591. https://doi.org/10.1177/0278364906065385

[30] Delphine Picard, Catherine Dacremont, Dominique Valentin, and Agn E Es Giboreau. [n. d.]. Perceptual dimensions of tactile textures. ([n. d.]). https: //doi.org/10.1016/j.actpsy.2003.08.001

[31] Early Reading, Ali Israr, Jill Fain Lehman, and Roberta L Klatzky. 2015. FeelSleeve : Haptic Feedback to Enhance. Proceedings of CHI 2015 (2015), 1015-1024.

[32] Catherine Reed, Roberta Klatzky, and Susan Lederman. 1989. Haptic Integration of Object Properties: Texture, Hardness, and Planar Contour. Fournal of Experimental Psychology (1989). http://scholarship.claremont.edu/cmc_fac_pub/406

[33] Luciano Santos and Carlos Vaz De Carvalho. 2013. Learning physics with virtua simulations. In Proceedings - 2013 2nd Experiment@ International Conference, exp.at 2013. IEEE, 184-185. https://doi.org/10.1109/ExpAt.2013.6703064

[34] Luciano Santos and Carlos Vaz de Carvalho. 2013. Haptic Physics Simulation. Proceedings of the European Conference on Games Based Learning 1 (2013), 771-775. http://connection.ebscohost.com/c/articles/ 91951045/haptic-physics-simulationhttp://jproxy.lib.ecu.edu/login?url=http: //search.ebscohost.com/login. aspx?direct=true \&db=ehh\&AN=91951045\&site= ehost-live

[35] David I. Shore, Kellie Gray, Emily Spry, and Charles Spence. 2005. Spatial modulation of tactile temporal-order judgments. Perception 34, 10 (2005), 1251-1262. https://doi.org/10.1068/p3313

[36] David I Shore, Emily Spry, and Charles Spence. 2002. Confusing the mind by crossing the hands. Cognitive Brain Research 14, 1 (2002), 153-163. https: //doi.org/10.1016/S0926-6410(02)00070-8

[37] Mads Solvsten Sorensen, Jesper Mosegaard, and Peter Trier. 2009. The visible ear simulator: a public PC application for GPU-accelerated haptic 3D simulation of ear surgery based on the visible ear data. Otology \& Neurotology 30, 4 (6 2009), 484-487. https://doi.org/10.1097/MAO.0b013e3181a5299b

[38] Georg von Békésy. 1960. Experiments in Hearing. The fournal of the Acoustical Society of America 88, 6 (1960), 2905. https://doi.org/10.1121/1.399656

[39] Katharina Weiß and Ingrid Scharlau. 2011. Simultaneity and temporal order perception: Different sides of the same coin? Evidence from a visual prior-entry study. The Quarterly fournal of Experimental Psychology 64, 2 (2 2011), 394-416. https://doi.org/10.1080/17470218.2010.495783

[40] Shinya Yamamoto and S Kitazawa. 2001. Reversal of subjective temporal order due to arm crossing. Nature Neuroscience 4, 7 (2001), 759-765. https://doi.org/10. 1038/89559

[41] Siyan Zhao, Ali Israr, Micah Fenner, and Roberta Klatzky. 2017. Intermanual Apparent Tactile Motion and its Extension to 3D Interactions. IEEE Transactions on Haptics 1412, c (2017), 1-1. https://doi.org/10.1109/TOH.2017.2678502

[42] Siyan Zhao, Ali Israr, and Roberta Klatzky. 2015. Intermanual apparent tactile motion on handheld tablets. IEEE World Haptics Conference, WHC 2015 (2015), 241-247. https://doi.org/10.1109/WHC.2015.7177720

[43] J. Zhou, X. Shen, and N D Georganas. 2004. Haptic tele-surgery simulation. In Proceedings. Second International Conference on Creating, Connecting and Collaborating through Computing. IEEE, 99-104. https://doi.org/10.1109/HAVE.2004. 1391889 